\title{
Knowledge, attitude and practice of pesticide use and serum cholinesterase levels among rice farmers in Nakhon Nayok Province, Thailand
}

\author{
Sapsatree Santaweesuk \\ Department of Public Health, Srinakharinwirot University, Nakhon Nayok, Thailand \\ Paisit Boonyakawee \\ Sirindhorn College of Public Health, Trang, Thailand, and \\ Wattasit Siriwong \\ College of Public Health Sciences, \\ Chulalongkorn University, Bangkok, Thailand
}

\begin{abstract}
Purpose - The study purposes were to investigate the knowledge, attitude and practice of pesticide use and to determine the levels of serum cholinesterase among rice farmers in Nakhon Nayok province, Thailand.

Design/methodology/approach - A cross-sectional study was carried out in communities in Nakhon Nayok Province, Thailand. Multistage random sampling was employed, selecting one person per each rice farmer household. Data was collected from 188 rice farmers using a structured face-to-face interview questionnaire. A reactive-paper finger-blood test was used to determine SChE levels. Chi-square test was used to determine the relationship between the practice of pesticide use and $\mathrm{SChE}$ levels.

Findings - The results indicated that the rice farmers had a fairly good knowledge and a neutral attitude toward the practice of pesticide use. Most of the respondents had a fairly proper practice of pesticide use. However, serum cholinesterase levels of the farmers were unsafe and risky. It signified that $68.1 \%$ of the farmers had a health risk from using pesticides. The prevalence of abnormal SChE levels among rice farmers with improper use was significantly higher than that among rice farmers who had a proper use.

Research limitations/implications - The result provided a guideline for operational planning and control and prevention of health effects from pesticides use in farmers or other agriculturists who use pesticides in cultivation.

Practical implications - Concerned local agencies especially health-related agencies are able to conduct a training to educate and build safety awareness including monitoring continuously the safety behaviors toward pesticides use. It can be implemented by establishing leaders from health promoting hospitals led by village health volunteers, community leaders and participation from farmers to collaboratively monitor proper use of pesticides beginning with health surveillance in the abnormal SChE group to be screened for pesticide exposure every six months or one year.

Social implications - Farmers gained knowledge and understanding of pesticides use and used it properly, which resulted in a reduction of pesticide residues in body as well as in the environment. In addition, the government policy should legislate measures for related agencies to promote proper use of pesticides. For instance, Department of Agriculture, Minister of Agriculture and Cooperatives should regularly supervise,
\end{abstract}

(C) Sapsatree Santaweesuk, Paisit Boonyakawee and Wattasit Siriwong. Published in Journal of Health Research. Published by Emerald Publishing Limited. This article is published under the Creative Commons Attribution (CC BY 4.0) license. Anyone may reproduce, distribute, translate and create derivative works of this article (for both commercial and non-commercial purposes), subject to full attribution to the original publication and authors. The full terms of this license may be seen at http:// creativecommons.org/licences/by/4.0/legalcode

This study was supported by the Faculty of Physical Education, Srinakharinwirot University, Thailand.

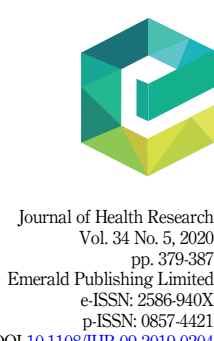

DOI 10.1108/JHR $09-2019-0204$

\section{Practice of pesticide use among rice farmers

Received 4 September 2019 Revised 12 November 2019 10 January 2020 Accepted 24 February 2020 
JHR 34,5 monitor and inspect the production and distribution of pesticides in local shops and also systematically encourage agriculturists to adopt using pesticides that are less harmful.

Originality/value - Having knowledge, understanding and proper practices toward pesticides use, farmers can reduce health effects of pesticides use in themselves and family members.

Keywords Agricultural safety and health, Rice farmer, Pesticide use, Serum cholinesterase

Paper type Research paper

\section{Introduction}

Pesticides are widely used for rice farming in Thailand. Statistics from 2007 to 2017 show that the import of pesticides into Thailand has been continuously increasing each year $(164,538$ tons in 2007 and 198,317 tons in 2017) [1]. Pesticides can be used freely by rice farmers, but cases of improper use can affect the health of the users, communities and the environment. As a result of inefficient pesticide use and management, people's long-term health can be affected. Prevalent symptoms are related to agricultural tasks in the paddy fields among rice farmers. The main symptoms experienced by farmers included a dry throat and cramp following the spraying and mixing of pesticides [2]. However, the reported illness data was lower than the actual data due to incomplete research and continuity of reporting [3].

Nakhon Nayok is located in the center of Thailand and divided into four districts.

Ongkharak is one of the districts with an efficient irrigation system which results in $3-5$

crops per year with rice farmers being in the majority of inhabitants [4]. In 2017, Ongkharak had a high ratio of farmers and the largest area with $33 \%$ of paddy fields in the province [5]. Being rice farmers, they are vulnerable to the effects of pesticide use, such as residue and biological magnification, which can cause many diseases. Previous studies in this area concerning pesticide use and the health effects of farmers found that farmers exposed to pesticides were significantly more likely to have adverse eye symptoms [6].

These health effects could be due to a lack of knowledge regarding the dangers of pesticide exposure and a negative attitude toward safety, in particular, the use of pesticides that result in the form of improper use. Therefore, this study aimed to investigate the knowledge, attitude and practice of pesticide use and determine the levels of serum cholinesterase among rice farmers in order to prevent and address the improper use of pesticides.

\section{Methods}

Study design

This cross-sectional study was conducted in Ongkharak district, Nakhon Nayok Province, Thailand, during the dry season from December 2017 to April 2018.

\section{Sample size and sampling technique}

The respondents were rice farmers in two communities (Ongkharak and Chumphon subdistrict). The inclusion criteria were rice farmers involved in all stages of rice cultivation: (1) land-preparing process, (2) seed-soaking and scattering/fertilizer-applying process, (3) pesticides-mixing and -spraying process, (4) sowing fertilizer and (5) rice-harvesting process. In addition, they had to be living in the study area for at least six months prior to the study and willing to participate in this research through informed consent. Farmers who were currently presenting with Alzheimer's disease, diabetes (non-insulin-dependent diabetes mellitus, type II), liver disease, kidney disease and taking brain injury medicine were excluded. A total of 188 respondents were calculated by the power and sample size calculations [7]. Multistage random sampling was employed by selecting one person from each rice farmer's household. 
Research instrument and data analysis

The data collected from rice farmers used a structured face-to-face interview questionnaire. The questionnaire was developed from previous studies $[8,9]$. There were a total of 12 closeended questions concerning knowledge of pesticides and attitudes related to pesticides, which had 12 items on a Likert scale of $1-5$ points (points were given for positive or negative direction questions). For the practice of pesticides, all respondents were interviewed with 12 questions face to face to choose only one from a four-point Likert scale, ranking with the frequency of practice: always, sometimes, rarely and never. The questionnaire was validated with pilot testing for reliability on 30 rice farmers who had similar characteristics to the respondents. KR-20 assessed the reliability of knowledge of pesticides, which was 0.71 , and the internal consistency reliability (Cronbach's $\alpha$ ) of attitude related to pesticide use and practice of pesticide use was 0.89 and 0.85 , respectively.

A reactive-paper finger-blood test was used to determine $\mathrm{SChE}$ levels. Blood collection in this process was performed by a registered nurse. A test kit was produced by the Government Pharmaceutical Organization, Thailand (sensitivity in field $=77.04 \%$, specificity in field $=90.01 \%$ and positive predicted value $=90.38 \%$ ) $[10]$. Four health risk levels were used to determine the magnitude of change in cholinesterase activities, as follows: normal ( $\geq 100$ units $/ \mathrm{ml})$, safe (87.5-99.9 units/ml), risky (75.0-87.4 units/ml) and unsafe $(<75.0$ units/ $\mathrm{ml}$ ) [10]. SPSS for Windows version 20 was used for statistical analysis. The descriptive statistics were analyzed for general information. A Chi-square test was used to determine the relationship between the practice of pesticide use and $\mathrm{SChE}$ levels.

\section{Ethical considerations}

This study was approved by the Review Boards of the Ethical Committee of Srinakharinwirot University, Thailand (No. SWUEC 066/60 E). All respondents received a clear explanation of the research procedures and their right to withdraw from the study at any time.

\section{Results}

General data

As shown in Table 1, the respondents were male (59.6\%) and female (40.4\%). The mean age was 54.7 years, and most of them were in the range of $50-59$ years old ( $30.8 \%)$. The majority of the respondents were married $(89.9 \%)$. Most of them graduated from primary school $(67.5 \%)$. The mean monthly family income was US\$ 428.4 (12,852 THB), and the average length of the current occupation was 28.6 years. The average work duration per day was $7.1 \mathrm{~h}$, with an average 15.5-acre (38.3 rais) farm size.

\section{Knowledge of the safe use of pesticides}

Knowledge of the safe use of pesticides was assessed by asking 12 questions. The results demonstrated in Table 2 show that the respondents had an average knowledge score of 8.7. It also illustrates that the majority of respondents $(47.9 \%)$ had a fairly good knowledge of the safe use of pesticides.

\section{Attitude related to pesticide use}

The majority of the respondents $(56.9 \%)$ had a neutral attitude toward pesticide use (Table 2 ). It is shown that when considering each question, almost all respondents agreed with the question "while spraying, you should stand in the direction of the wind." On the other hand, many respondents agreed with negative questions such as "you can smoke or eat while spraying pesticide."

\section{Practice of pesticide use among rice farmers}




\section{Gender}

Male

Female

Age group (years)

382

$\leq 29$

40-49

Mean $\pm S D=54.71 \pm 11.64$, Min. - Max. $=18-68$

Marital status

Married

Single/widowed/divorced/separated

\section{Education}

Never attended school 2

Primary school 127

Secondary school

Equal to or higher than high school

Smoking cigarette

Yes

No

Characteristics

Monthly family income (US\$*)

Work duration per day (hour)

Note(s): $* 1$ US $\$$ was approximately 30 Thai baht, $* * 1$ acre $=2.47$ rai

Variable

Frequency $(n=188)$

Knowledge of the safe use of pesticides (point)

Low (2-5)

Moderate (6-8)

High (9-11)

Mean $\pm S D=8.7 \pm 1.8$, Min. - Max. $=2-11$

Attitude related to pesticide use (point)

Negative (14-29) 42

Table 2.

Frequency and percentage of knowledge on the safe use of pesticides, attitude related to pesticide use and practice of pesticide use
Positive (46-60)

Mean $\pm S D=42.1 \pm 8.3$, Min. - Max. $=14-60$

Practice of pesticide use (point)

Low (12-19)

Moderate (20-27)

High (28-36)

Mean $\pm S D=26.3 \pm 7.6$, Min. - Max. $=12-36$ 
Practice of pesticide use

Table 2 shows that most respondents $(46.6 \%)$ had a fairly proper practice of pesticide use. When considering the questionnaire responses concerning the frequency of practices, it was found that the most frequent acts performed during pesticide use among respondents were as follows: exploring the area before spraying pesticide ( $64.3 \%)$, being more careful when using a pesticide $(65.0 \%)$ and in cases of exposure, drenching the area with water immediately $(56.6 \%)$. Moreover, in terms of personal hygiene, most of them $(59.1 \%)$ cleaned themselves immediately after using pesticides. However, there were still some practices identified that need to be improved since some respondents still practiced improper use of pesticides. For instance, in cases of pesticide use and its storage, $20.4 \%$ of respondents did not store pesticides in the original spot after use. Or in cases of pesticide protection, 30.6\% used improper personal protective equipment. In addition, some improper uses were sometimes present in a relatively high proportion, for example, mixing pesticides with more than one kind, drinking while spraying pesticide, after mixing pesticides, not keeping it in its original package, not changing clothes after returning home and not separating work clothes from other clothes. If there is no improvement, there is a greater health risk to users.

\section{Serum cholinesterase level (SChE)}

Table 3 shows that the prevalence of abnormal SChE levels was $68.1 \%(38.3 \%$ unsafe and $29.8 \%$ risky) and the remainder was normal ( $3.7 \%$ normal and $28.2 \%$ safe). Table 4 shows the practice of pesticide use related to the $\mathrm{SChE}$ level. The prevalence of abnormal SChE levels among rice farmers with improper use was significantly higher than rice farmers who practiced proper use.

\section{Discussion}

The knowledge of rice farmers was found to be good and confirms results from a previous study where farmers possessed knowledge of pesticide as high as $88.7 \%$ [11]. The reason could be that most of the farmers have practiced farming for a long time, many with an average period of 28.6 years, and therefore, may have gained knowledge of pesticide use through the media, for example, television, public health officers and village health volunteers. However, $22.3 \%$ of the farmers still had incorrect information concerning some

\begin{tabular}{lccr}
\hline Health risk level & (SChE) level (units/ml) & Frequency $(n=188)$ & $(\%)$ \\
\hline Normal & $\geq 100$ & 7 & 3.7 \\
Safe & $87.5-99.9$ & 53 & 28.2 \\
Risky & $75.0-87.4$ & 56 & 29.8 \\
Unsafe & $<75.0$ & 72 & 38.3
\end{tabular}

Practice of pesticide use among rice farmers

383

\begin{tabular}{lcccccr}
\hline Practice in & $\begin{array}{c}\text { Normal } \\
\text { pesticide use }\end{array}$ & $\begin{array}{c}\text { Safe }(n=53) \\
(\%)\end{array}$ & $\begin{array}{c}\text { Risky } \\
(n=56)(\%)\end{array}$ & $\begin{array}{c}\text { Unsafe } \\
(n=72)(\%)\end{array}$ & $\begin{array}{c}\text { Total } \\
(n=188) \\
(\%)\end{array}$ & $p$-value \\
\hline Low & $1(14.3)$ & $3(5.7)$ & $17(30.4)$ & $22(30.6)$ & $43(22.9)$ & $<0.001^{*}$ \\
Moderate & $2(28.6)$ & $18(33.9)$ & $26(46.4)$ & $42(58.3)$ & $88(46.6)$ & \\
High & $4(57.1)$ & $32(60.4)$ & $13(23.2)$ & $8(11.1)$ & $57(30.5)$ &
\end{tabular}

Note(s) : Fisher's Exact Test

\section{Table 3 . \\ Frequency and percentage of serum cholinesterase level (SChE)}

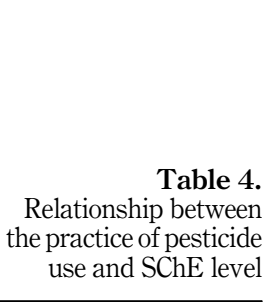


JHR 34,5

\section{4}

pesticide use, for example, farmers believed that some pesticides, such as paraquat, were not hazardous to its users. Hence, they thought it was unnecessary to wear a respirator during the pesticide-spraying process. Furthermore, in this study, some farmers still misunderstood that pesticides could only enter the body through inhalation. This was similar to the previous study, which found that farmers were still displaying a lack of knowledge and understanding that pesticides can enter the human body in various ways; consequently, they may also lack protection while using pesticides [12].

The study indicated that the respondents' attitudes to pesticide use were at a neutral level, accounting for $56.9 \%$. This may be because the farmers had accurate information that in turn affected their attitude toward pesticide use [12]. Nevertheless, there still were some farmers who had a negative attitude toward the use of PPE (personal protective equipment). Most of them thought that using PPE was an inconvenience while working and that wearing PPE made them feel uncomfortable and too hot. In addition, it was found that farmers had an attitude that "if you are allergic to pesticides, your body can recover from allergies without having to see a doctor." This resulted in them tending to neglect their health leading in turn to chronic health problems. This is per the result of Boonyakawee et al. [9], who found that intervention programs including special training could adjust attitudes about pesticide use in a positive way. Concerned public health agencies should, therefore, implement safety awareness in the form of various programs to its local farmers to create a positive attitude toward safe pesticide use.

The practice of pesticide use in rice farming was at a moderate level, with examples of proper practice and improper use of pesticides. Most rice farmers stated that they would be more cautious of potential dangers when using pesticides compared to working in other processes. For example, when chemicals splashed onto the body, they would immediately clean it. Moreover, they used PPE while spraying pesticides. Yet, the quality of their PPE did not always protect them from the dangers of pesticides at maximum efficiencies, such as wearing and wrapping clothes over their heads and noses. As shown in a study of farmers in Uganda, the main problem was that they did not use proper PPE, which resulted in toxic poisoning from pesticides [13]. This finding is also in line with the study about health risk assessment of farmers in Nakhon Nayok province, Thailand, which found farmers were at risk of adverse health effects due to dermal exposure to chlorpyrifos from the improper use of PPE [14]. In addition, some farmers still performed risky practices of getting pesticides into the body, that is, drinking and eating in the area where spraying was carried out. There were still chemical residues in the environment that could enter the human body.

Serum cholinesterase (SChE) levels of the farmers were unsafe and risky (38.3\% and $29.8 \%$, respectively) and signified that $68.1 \%$ of the farmers had health risks from using pesticides. It was also found in a study of cholinesterase blood tests of agriculturists that most agriculturists were rice farmers and had a risky SChE level at $72.2 \%$ and only $3.7 \%$ of rice farmers had normal SChE levels [15]. This result is consistent with a study about pesticide exposure and cholinesterase levels in migrant farm workers in Thailand, which found that only $4.4 \%$ of farm workers had normal levels of cholinesterase activity and $58.5 \%$ were at risk [16]. Likewise, in cases of having pesticide residues that persisted for a long time, this contributed to health problems referred to in the study on serum cholinesterase levels of Thai chili-farm workers who were exposed to chemical pesticides. It found that the prevalence of pesticide-related symptoms among farmers with abnormal SChE levels was significantly higher than that among the normal group [17]. This study also found that the prevalence of abnormal SChE levels among rice farmers with improper use was significantly higher than that among rice farmers who followed proper usage practices. A finding consistent with Boonyakawee $e$ t al. [18] was that farmers who usually used PPE had better SChE levels than those who did not. 
With regard to personal factors and socioeconomic factors that might affect the $\mathrm{SChE}$ levels, it was found in a study by Chaiklieng and Praengkrathok [19] that there was no statistical relationship between factors as gender, age, education, income, pesticide exposure duration, social support and SChE levels. Likewise, a study on SChE levels of Thai Chilli-farm workers also found that age was not relevant to abnormal SChE levels [17]. However, there was a study on factors associated with the farmers' blood cholinesterase level, which showed the statistical significance between gender and SChE levels [20]. This may be because females are weaker in physique and have less strength than males. Therefore, when performing work that is at risk of chemical exposure, females have higher levels of pesticide residues in the body than males.

Knowledge and attitude toward pesticides are mutually connected and linked to the practice of pesticides. Improper use of pesticides was a risk factor for health issues. The results of this study indicated that rice farmers were at risk of receiving pesticide residues into the body, which could be the result of a lack of understanding by farmers toward pesticide use. In addition, rice farmers are only concerned with chemical substances that can enter the body through inhalation. This could consequently lead to a lack of protection against pesticides through other exposure pathways and affect the body. Thus, similar to the study on pesticide residue, it was discovered that pesticide residues were detected on the dermal patches, face wipes and hand wipes of respondents. Moreover, the main relationship between pesticide exposure and urinary metabolite was found to have been relevant to dermal exposure [21]. In the event of a lack of realization of farmers that chemical substances can be absorbed into the body through dermal exposure, they may not cleanse their body immediately after the use of pesticides and it can, therefore, be residual in their body. Thus, training and supervising health professionals to provide information on safe use and other standard information about pesticides for rice farmers should be introduced in the area.

One limitation of this study should be noted. This study assessed only the abnormal SChE levels among rice farmers and improper use of pesticides. There were no other potential variables to be analyzed. Thus, the results may not effectively conclude the association. Moreover, a reactive-paper finger-blood test was used to screen the risk of farmers exposed to organophosphates (OPs) and carbamates pesticides, which are insecticides commonly used by rice farmers [2]. In addition to the mentioned two types of chemicals that affect cholinesterase, it was found that some substances influenced cholinesterase, for example, medicines for Alzheimer's disease and brain injury medicine [10]. Nevertheless, this type of testing entailed uncertain test results in patients with diabetes or kidney disease patients because these patients have lower levels of cholinesterase [20]. These factors are assumed to affect the interpretation of the screening test results. Hence, there should be requirements for specific cases before and after the screening test. In this study, we conducted interviews on underlying disorders and medication to help with the interpretation of results to prevent discrepancies due to the mentioned factors.

The recommendations for rice farmers being screened using reactive-paper finger-blood test to change behaviors of pesticide use according to levels of risk are as follows: 1 ) if the test results are normal or safe, surveillance by drawing blood once a year and keeping healthy according to general healthcare principles must be performed, with the main purpose of making this group of farmers healthy and not to be a risky or unsafe group. 2) if the rice farmers acquire the level of risky or unsafe levels, they must be followed up every 30 days until the test results are normal or safe. After that, surveillance must be carried out by drawing blood every six months. In addition, public health officers should suggest that the farmers stop being exposed to chemicals by changing jobs and should return to work once the blood test results are at a normal or safe level. Furthermore, local public health agencies should collaboratively work with local leaders and community leaders including nearby academic institutions by focusing on changing behavior through the cooperation of people in
Practice of pesticide use among rice farmers

385 
the community to create understanding and raise awareness of health and environmental effects of improper pesticide use.

\section{Conclusion}

It is recommended that the local authorities concerned, especially the local health organization, should promote positive attitudes, such as the benefits of effective use of correct PPE, and knowledge toward the safe use of pesticides to farmers along with awareness-raising programs and regular monitoring of the safe use of pesticides. Mainstays from health-promoting hospitals led by village health volunteers, community leaders and the participation of farmers should be established to cooperatively monitor the proper use of pesticides. The policy on regular monitoring of pesticide exposure should be developed by the national authorities concerned. For instance, the Ministry of Public Health and the Ministry of Agriculture and Cooperatives should regularly supervise, monitor and inspect the production and distribution of pesticides in local shops and also systematically encourage agriculturists to adopt using less harmful pesticides.

Future studies should focus on the health effects of using pesticides in vulnerable groups and family members, including programs that can sustainably reduce the health risks from pesticide use among farmers, family members and communities. This study was carried out among rice farmers in the agricultural sector. The findings can be generalized reasonably to rice farmers who have similar characteristics, especially in the central region of Thailand.

\section{References}

1. Office of Agricultural Economics. Quantity and cost of pesticide imports to Thailand during 20112017. [cited 2018 April 2]. Available at: http://www.oae.go.th/view/1//TH-TH.

2. Sapbamrer R, Nata S. Health symptoms related to pesticide exposure and agricultural tasks among rice farmers from Northern Thailand. Environ Health Prev Med. 2014 Jan; 19(1): 12-20. doi: 10.1007/s12199-013-0349-3.

3. Bureau of Occupational and Environmental Diseases. Ratio of pesticide poisoning 2010-2013. [cited 2016 Jan 23]. Available at: http://envocc.ddc.moph.go.th/contents/view/106.

4. Pathum Thani Rice Research Center. Knowledge management corner. [cited 2016 June 12]. Available at: https:/www.brrd.in.th/rkb/.

5. Ongkharak Agricultural Extension Office. Agricultural information in Ongkharak district. [cited 2017 May 7]. Available at: http://ongkharak. nakhonnayok.doae.go.th/.

6. Wilaiwan W, Siriwong W. Assessment of health effects related to organophosphate pesticides exposure using blood cholinesterase activity as a biomarker in agricultural area at Nakhon Nayok province, Thailand. J Health Res. 2014; 28(1): 23-30.

7. Dupont WD, Plummer WD, Jr Power and sample size calculations: a review and computer program. Control Clin Trials. 1990 Apr; 11(2): 116-128.

8. Santaweesuk S, Chapman RS, Siriwong W. Effects of an injury and illness prevention program on occupational safety behaviors among rice farmers in Nakhon Nayok Province, Thailand. Risk Manag Healthc Policy. 2014; 7: 51-60. doi: 10.2147/rmhp.S55810.

9. Boonyakawee P, Taneepanichskul S, Chapman RS. Effects of an intervention to reduce insecticide exposure on insecticide-related knowledge and attitude: a quasi-experimental study in Shogun orange farmers in Krabi Province, Thailand. Risk Manag Healthc Policy. 2013; 6: 33-41. doi: 10. 2147/RMHP.S50409.

10. Bureau of Occupational and Environmental Diseases. Methods of screening for risk of pesticide exposure by cholinesterase reactive paper. Department of Disease Control, Ministry of Public Health; 2015, Nonthaburi. 
11. Tritipsombut J, Gabklang P, Boonkerd S, Oapsuwan A. The study of knowledge, attitudes and pesticide usage behaviors among the agricultural workers at Huay Sam Kha village, Tub Ruang sub-district, Phra Thong Kum district, Nakhon Ratchasima province. Srinagarind Med J. 2014; 29(5): 429-34.

12. Gesesew HA, Woldemichael K, Massa D, Mwanri L. Farmers knowledge, attitudes, practices and health problems associated with pesticide use in rural irrigation villages, southwest Ethiopia. PLoS One. 2016; 11(9): e0162527. doi: 10.1371/journal.pone.0162527.

13. Oesterlund AH, Thomsen JF, Sekimpi DK, Maziina J, Racheal A, Jors E. Pesticide knowledge, practice and attitude and how it affects the health of small-scale farmers in Uganda: a crosssectional study. Afr Health Sci. 2014 Jun; 14(2): 420-33. doi: 10.4314/ahs.v14i2.19.

14. Lappharat S, Siriwong W, Taneepanichskul N, Borjan M, Maldonado Perez H, Robson M. Health risk assessment related to dermal exposure of chlorpyrifos: a case study of rice growing farmers in Nakhon Nayok Province, Central Thailand. J Agromedicine. 2014; 19(3): 294-302. doi: 10.1080/ 1059924X.2014.916643.

15. Pengput A, Pramual P. Factors association with the risk of pesticide residual by cholinesterase blood test of agriculturists in Songplueay sub-district, Namon district, Kalasin province. KKU Journal for Public Health Research. 2017; 10(1): 47-62.

16. Thetkathuek A, Yenjai P, Jaidee W, Jaidee P, Sriprapat P. Pesticide exposure and cholinesterase levels in migrant farm workers in Thailand. J Agromedicine. 2017; 22(2): 118-130. doi: 10.1080/ 1059924X.2017.1283276.

17. Kachaiyaphum P, Howteerakul N, Sujirarat D, Siri S, Suwannapong N. Serum cholinesterase levels of Thai chilli-farm workers exposed to chemical pesticides: prevalence estimates and associated factors. J Occup Health. 2010; 52(1): 89-98. doi: 10.1539/joh.q9003.

18. Boonyakawee P, Taneepanichskul S, Chapman RS. Knowledge, attitude and practices in insecticide use, serum cholinesterase levels and symptom prevalences among Shogun orange farmers in Khao-Phanom district, Krabi province Thailand, 2012. J Health Res. 2013; 27(3): 191-6.

19. Chaiklieng S, Praengkrathok S. Risk assessment on pesticide exposure by biological monitoring among farmers: a case study in Tambon Kangsanamnang, Nakhonratchasima province. Srinagarind Med J. 2013; 28(3): 382-9.

20. Chuenchom T. Factors associated with the farmers' blood cholinesterase level. Region 4-5 Medical Journal. 2018; 37(2): 86-97.

21. Taneepanichskul N, Norkaew S, Siriwong W, Siripattanakul-Ratpukdi S, Maldonado Perez HL, Robson MG. Organophosphate pesticide exposure and dialkyl phosphate urinary metabolites among chili farmers in northeastern Thailand. Rocz Panstw Zakl Hig. 2014; 65(4): 291-9.

\section{Corresponding author}

Sapsatree Santaweesuk can be contacted at: sapsatreejournal@gmail.com
Practice of pesticide use among rice farmers 